\section{Site Specific Three-dimensional Structural Analysis in Tissues and Cells Using Automated DualBeam Slice \& View}

\author{
Ben Lich \\ FEI Company, Eindhoven, The Netherlands \\ Ben.Lich@fei.com
}

\section{Introduction}

DualBeam instruments that combine the imaging capability of scanning electron microscopy (SEM) with the cutting and deposition capability of a focused ion beam (FIB) provide biologists with a powerful tool for investigating three-dimensional structure with nanoscale $(1 \mathrm{~nm}-100 \mathrm{~nm})$ resolution. Ever since Van Leeuwenhoek used the first microscope to describe bacteria more than 300 years ago, microscopy has played a central role in scientists' efforts to understand biological systems. Light microscopy is generally limited to a useful resolution of about a micrometer. More recently the use of confocal and electron microscopy has enabled investigations at higher resolution. Used with fluorescent markers, confocal microscopy can detect and localize molecular scale features, but its imaging resolution is still limited. SEM is capable of nanometer resolution, but is limited to the near surface region of the sample. Transmission electron microscopy (TEM) can provide sub-Angstrom resolution in some materials, though not generally in biological applications. TEM-based electron tomography is playing an increasingly important role filling the critical imaging gap between cellular and atomic scales, especially in determining the tertiary and quaternary structure of proteins, protein complexes and other biological macromolecules.

DualBeam instruments offer an attractive alternative for nanoscale imaging and three-dimensional structural investigations. The DualBeam technique, known as "slice and view", consists of acquiring a sequence of cross sectional images spaced evenly through a region of a bulk specimen, and reconstructing those two-dimensional images into a three-dimensional representation of the sampled volume. It has been used successfully on tissues and whole cells, and is particularly well-suited for composite specimens that include both hard and soft components. It also provides an effective method for preparing thin sections for TEM imaging and analysis.

\section{DualBeam Configuration}

FIB and SEM have been used for decades in materials science applications, and over the most recent decade, have become an indispensable component of semiconductor manufacturing operations where their ability to provide precisely positioned cross sections through nanoscale structures enables tight control of the manufacturing process. The combination of FIB and SEM in a single instrument is an excellent example of the whole being much greater than the sum of its parts. In DualBeam instruments (FEI Company, Hillsboro, Oregon, USA), the ion beam and the electron beam focus and intersect at a point on the sample surface known as the coincident point - the electron beam is vertical and the ion beam
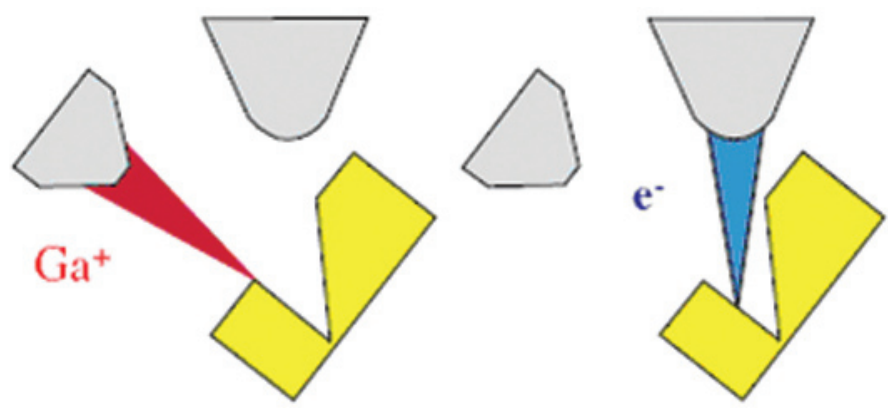

Figure 1: In DualBeam instruments, the ion and electron columns are configured so that their beams focus and intersect at the sample surface. The Ga+ ions of the ion beam are used to cut site specific cross sections that reveal subsurface structure. The electron beam provides immediate high-resolution imaging of the freshly-exposed cross-sectional surface. ${ }^{l}$

is off vertical by about 50 degrees (fig 1). With the sample tilted normal to the ion beam, ion milling creates a cross sectional surface that can be immediately imaged at high resolution by the electron beam without moving the sample. This permits close control of the milling process, ensuring that it is stopped when it reaches the feature of interest-before it destroys the very information it seeks.

\section{FIB}

The massive, high-energy ions $(\mathrm{Ga}+)$ of the FIB remove material by sputtering atoms and molecules from the sample surface. Their confinement in a tightly-focused beam provides precise spatial control of the milling process. FIB cross sectioning has several advantages over mechanical sectioning techniques. Knife marks and other cutting artifacts are largely eliminated. FIB milling performs particularly well on samples that include both hard and soft materials that would tend to tear or smear when cut by mechanical means. In addition to its milling capability, FIB can also be used to deposit material. This is accomplished using a chemical vapor deposition process in which the gas precursor is injected into the vicinity of the beam and the sample surface through a retractable needle. Interaction with the beam decomposes the precursor gas into a volatile component that is pumped away by the vacuum system and a non-volatile component that forms a deposit on the sample surface. Deposition is often used to apply a metal coating prior to cross sectioning to protect the sample from unintentional milling that could destroy critical near-surface detail. Deposition may also be used to create structures or attach pieces of the sample to external probes. Beam induced gas reactions may also be used to selectively enhance etch rates on certain types of material. Multiple injectors and a variety of gases allow enhanced etching or deposition of different materials.
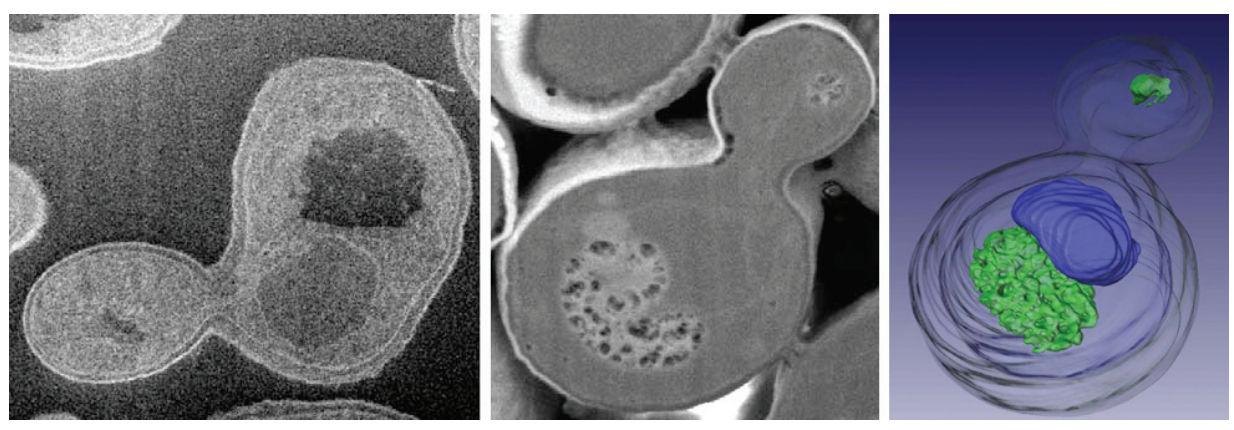

Figure 2 Budding yeast cells. Image courtesy of Sriram Subramaniam, NCI, NIH Bethesda, US ${ }^{1}$ 


\section{FEI COMPANY"}

TOOLS FOR

\section{The world's most powerful analytical FEG/SEM ever}

FEl's Quanta ${ }^{\mathrm{TM}}$ 3D FEG is the most versatile high resolution low vacuum FEG/SEM ever for 3D material characterization. With its innovative ion and electron optics it delivers superior results for FIB milling as well as SEM imaging and microanalysis, making it the ultimate analytical solution for materials research.

Unmatched high current FIB capability enables very fast milling to reveal sub-surface structures and features. Increased electron beam current enhances EDS and EBSP analytical results. A novel electron source design gives improved SEM imaging. Combining those enhancements with Quanta's unique environmental SEM operating mode results in a very powerful solution for investigation of hydrated, heated, and other samples that are difficult to analyze using conventional FEG/SEM systems.

For more information: www.fei.com/Quanta3Dfeg5 


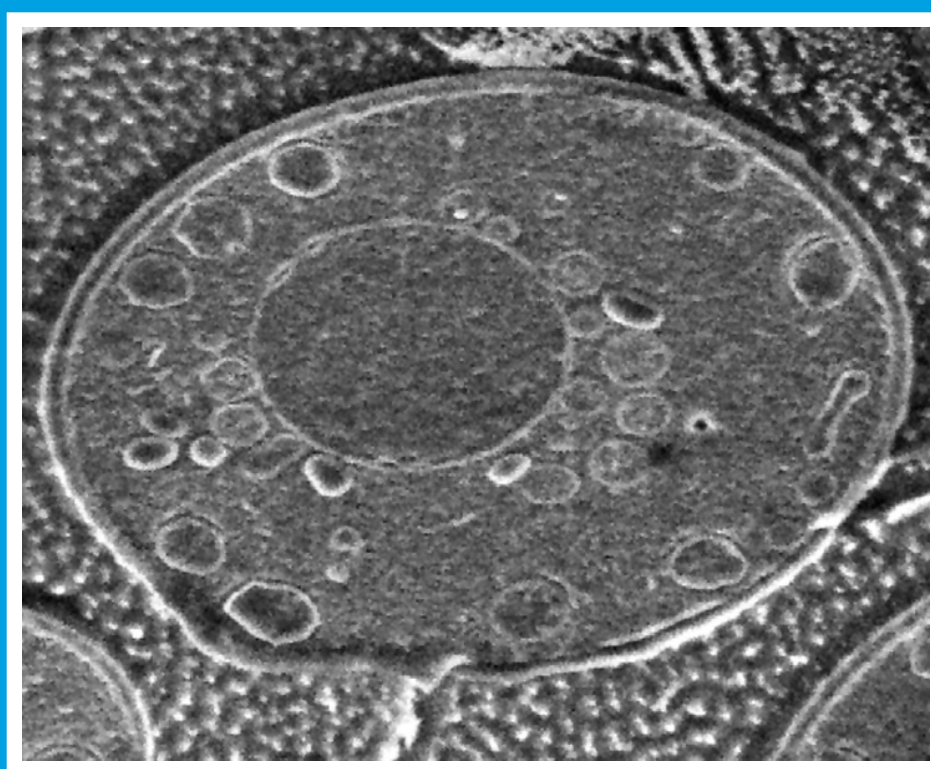

Figure 3: Plunge frozen yeast cell cross-sectioned with FIB. Image courtesy of Sriram Subramaniam, NCI, NIH Bethesda, US ${ }^{1}$

Although the primary use of FIB in biological applications is likely to be cutting cross sections and depositing protective layers, its imaging capabilities should not be neglected. The beam ions create a number of useful signals when they impact the sample, including secondary ions and secondary electrons. Because the ions are large, they penetrate less deeply than electrons. In situations where electron imaging resolution is limited by excessive penetration, or where surface specific information is sought, ion imaging may be a better choice. Ion images often contain information that is complementary to electron images. Current-generation DualBeam systems can provide FIB images with resolution better than $5 \mathrm{~nm}$.

FIB images can suffer from charging artifacts similar to those encountered in SEM images, though the accumulated charge is usually positive rather than negative. In DualBeam instruments, the electron beam can be used to provide low energy electrons for charge neutralization.

\section{SEM}

The primary advantage of SEM is improved image resolution. Current-generation DualBeam instruments offer SEM resolution better than $1 \mathrm{~nm}$. Though this is typically not achievable on biological specimens, order of magnitude improvements over optical resolution are routine. Generating sufficient contrast in biological materials can also be challenging. Over the years biologists have developed a range of staining techniques to enhance contrast, often involving the use of heavy metals. These are very
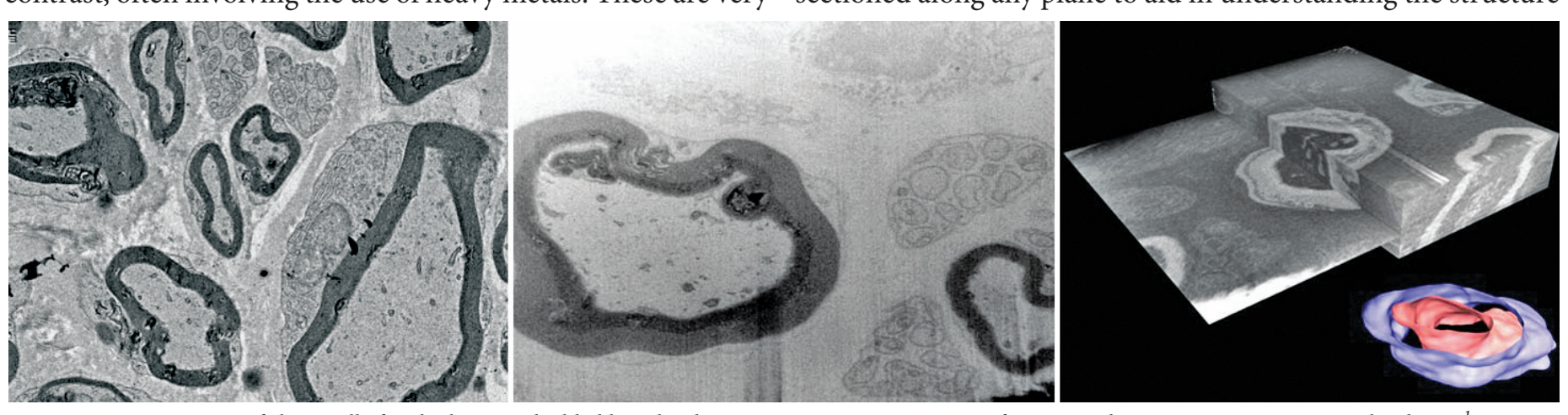

Figure 4 Images of chemically fixed, plastic embedded lymphoid tumor tissue. Image courtesy of Sriram Subramaniam, NCI, NIH Bethesda, US ${ }^{1}$ effective for creating amplitude contrast in TEM images, and also can be used for Backscatter imaging in an SEM or DualBeam. Although biologists have developed an extensive body of sample preparation techniques including procedures for fixing, embedding and staining a wide range of biological materials, they, more than most microscopists, would like to investigate their samples in a pristine natural state. Cryo EM on vitrified samples offers just that possibility. In vitrification, the freezing process occurs expanded crystalline matrix that is so destructive to biological microstructure.

SEM imaging on non-conductive material, like resin embedded Biopsies, is subject to charging artifacts as charge deposited by beam electrons exceeds charge escaping as secondary electrons. The secondary electron yield is a function of beam energy. As the energy is reduced, beam electrons penetrate less deeply and create more secondaries close enough to the surface to escape. At some voltage the escaping charge just balances the deposited charge and charging is minimized. Historically, electron columns have had relatively poor resolution at low accelerating voltages, forcing a trade-off between resolution and charging. Current-generation DualBeams offer excellent low-voltage performance, making low-voltage operation a viable method for charge control.

An alternative approach involves imaging at lower vacuum (higher pressure) than conventional SEM. In low vacuum conditions, positive ions created by the ion beam from the ambient gas are available to neutralize any negative charge that accumulates at the sample. Today, DualBeam systems are available with low vacuum electron imaging capability, though the ion beam must always operate under high vacuum conditions.

\section{Slice and View}

The slice and view process begins by the milling of a wedgeshaped trench in the sample. One wall of the trench is vertical (i.e., normal to the specimen surface) and becomes the initial cross section imaged by the electron beam. After imaging, the ion beam is used to remove a uniform layer of material from this wall, advancing the cross section a predetermined distance through the sample volume. Another electron image is collected and the milling/imaging process iterates until the cross section advances through the targeted volume. The sequence of images acquired at each step is then combined to construct a threedimensional representation of the sectioned volume. A flexible rendering engine permits intuitive visualization of the data set. The rendered model can be rotated to any viewing angle and resectioned along any plane to aid in understanding the structure so rapidly that water molecules do not have time to form the 


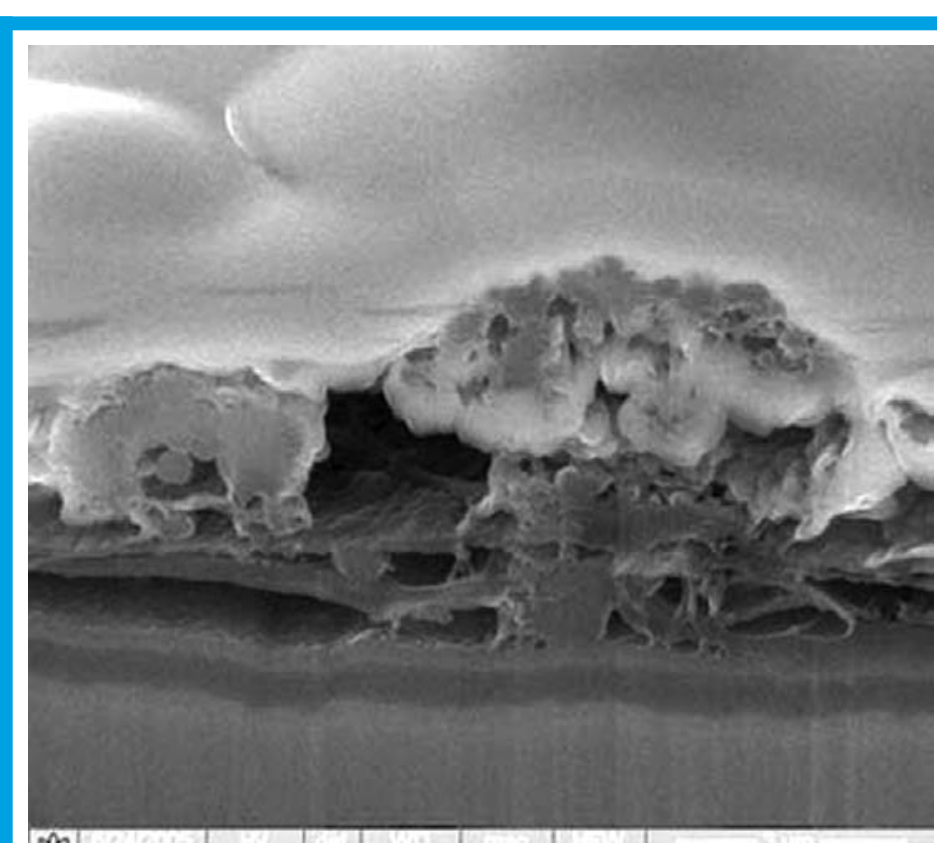

$\$$

Figure 5 SEM image of a FIB milled cross section through soft bone cells grown on a hard substrate (hydroxyapatite-coated titanium). Image courtesy of D.J. Stokes of the Cavendish Laboratory at the University of Cambridge $^{2}$

it represents. Automation provides for unattended execution of the entire cross sectioning and imaging procedure.

\section{Applications}

Cells

SEM images (Figure 2, left and middle) of budding yeast cells sectioned by FIB. The cell on the left was plastic-embedded. The one in the middle was critical point dried. The fig 2 image on the right shows a three-dimensional visualization of a plastic embedded yeast cell created using the slice and view method, and rendered to show the cell wall as a grey envelope, the nucleus in blue and the vacuolar region in green. In figure 3 we see an SEM image of a plunge frozen yeast cell cross-sectioned with FIB. Surface topography was enhanced by allowing the temperature of the sample to rise briefly to permit sublimation, followed by coating with platinum-palladium. Note the nuclear pore observable at approximately the 10 oclock position on the nuclear membrane.

\section{Tissues}

Figure 4 Shows a TEM image (left) and SEM image (middle) of chemically fixed, plastic embedded lymphoid tumor tissue. The TEM section was cut with a microtome. The SEM section was FIB milled. Contrast is inverted in the SEM image to facilitate comparison. The right most image shows a three-dimensional representation created with the DualBeam slice and view method. The inset image is the central feature of the image stack rendered with density thresholding.

\section{Soft/Hard Composites}

Figure 5 is an SEM image of a FIB milled cross section through soft bone cells grown on a hard substrate (hydroxyapatite-coated titanium). The white material at the top of the image is platinum metal deposited by FIB-induced CVD to protect the delicate bone cells from unintentional milling during the cross sectioning process.

\section{TEM lift-out}

Figure 6 Illustrates how a DualBeam can be used to prepare site specific thin sections for high resolution TEM imaging and analysis. The image on the left shows a thick section, ready for extraction, created by FIB milling material from both sides of the targeted structure. It has been attached to a micromanipulator probe with material deposited by beam-induced CVD. After extraction it will be attached to a grid and further thinned to electron transparency. The grid/sample is then transferred to a S/TEM to acquire a tomographic image series as it is rotated incrementally about an axis perpendicular to the beam. The middle image shows a TEM image acquired after final thinning. The right image shows a slice through the 3D model reconstructed from the tomographic images of a region in the extracted section.

\section{Cylindrical tomography sample}

Figure 7 Makes the point that tomography images acquired from flat thin sections are incomplete since projection images cannot be acquired from a range of directions lying close to the plane of the sample. Cylindrical specimens may offer a solution to this problem since they can be rotated and imaged through a full 360 degree range with no change in the electron path length through the specimen. Computer-controlled FIB milling can create arbitrarily complex shapes, including cylinders. The left
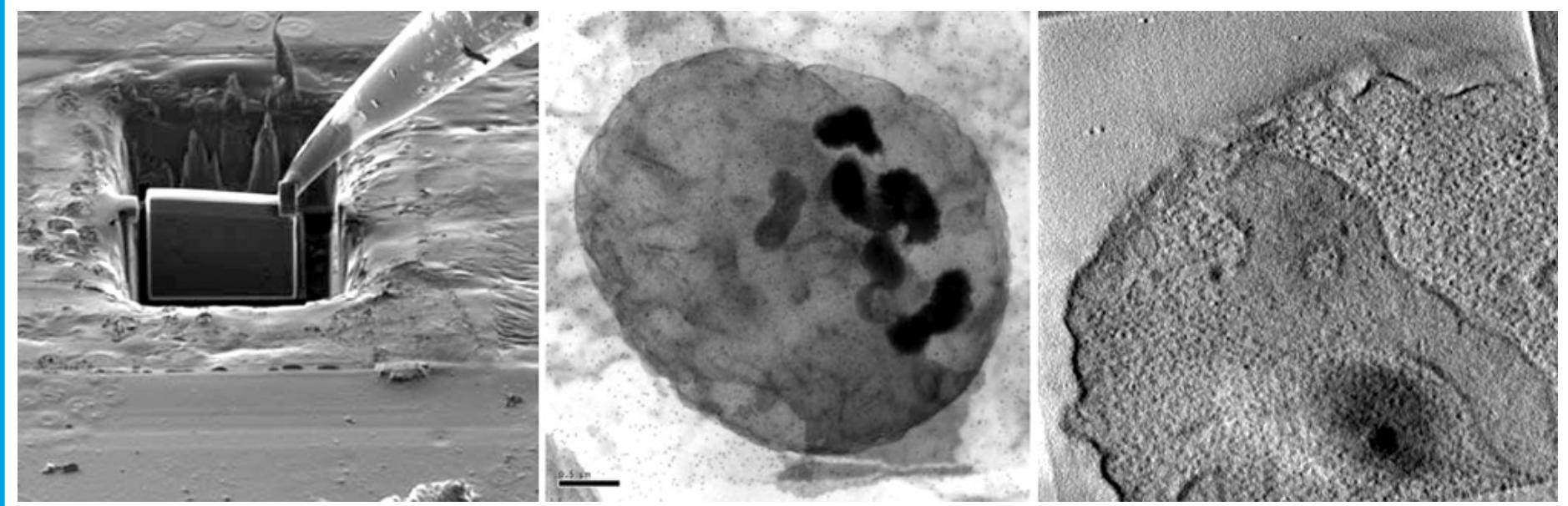

Figure 6 DualBeam used to prepare site specific thin sections for high resolution TEM imaging and analysis. Image courtesy of Sriram Subramaniam, NCI, NIH Bethesda, US ${ }^{1}$ 


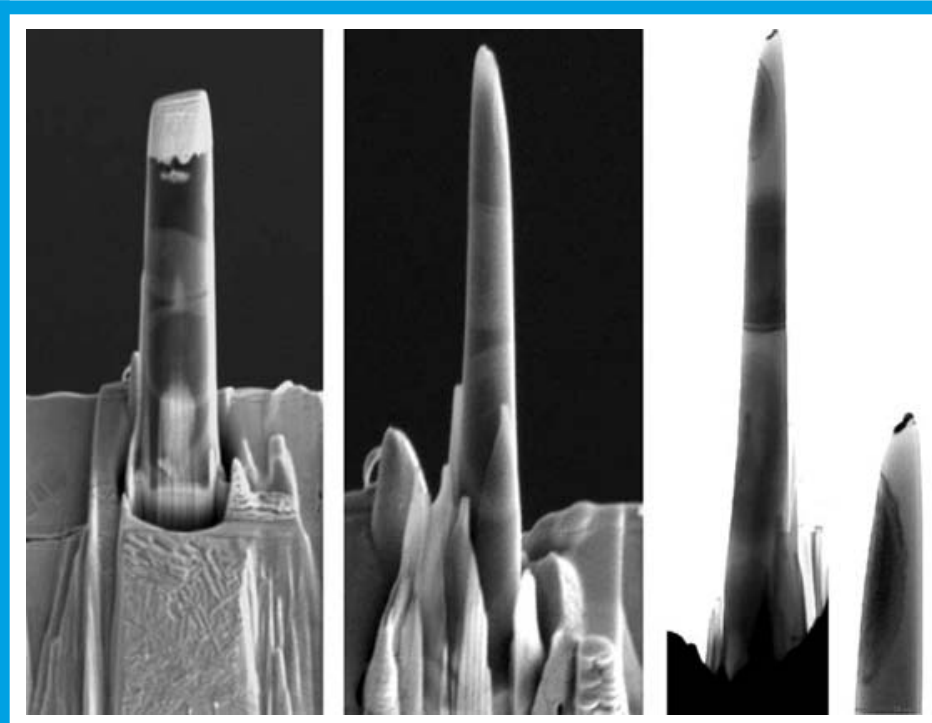

Figure 7 Cylindrical specimens prepared by DualBeam to facilitate TEM tomagraphy analysis. Specimens have a constant thickness independent of tilt angle with respect to the beam. Image courtesy of Sriram Subramaniam, NCI, NIH Bethesda, US ${ }^{1}$

and middle left images are SEM images of progressive stages in the milling of a cylindrical shape as the diameter is gradually reduced. The platinum at the tip of the specimen prevents excessive milling there. The middle right and right images are TEM images (at lower and higer magnifications respectively) of the same specimen when milling is complete. The central region is nearly cylindrical and less than a micrometer in diameter. A portion of a plastic embedded yeast cell is visible to the left side of the shaft just below the tip.

\section{Conclusion}

DualBeam slice and view is a powerful tool for visualizing three dimensional structures in biological specimens with resolution at least an order of magnitude better than optical microscopy. It reduces or eliminates many of the artifacts associated with mechanical sectioning and is particularly well suited for composite specimens that contain both hard and soft materials. Combined with cryo EM and sample vitrification technology, it can be used to visualize structure in a pristine natural condition. DualBeam is also expected to play a growing role in the creation of thin sections for S/TEM analysis.

\section{Acknowledgements}

The author wishes to thank Sriram Subrmaniam and Jurgen Heymann of the Labortory of Cell Biology at the National Cancer Institute (NIH, Bethesda, MD, USA) and D.J. Stokes of the Cavendish Laboratory at the University of Cambridge (Dept of Physics, Madingley Road, Cambridge, CB3 OHE, UK) for the images used in this article.

\section{References}

1) Heymann et al, Site Specific 3D Imaging of Cells and Tissues With a Dual Beam Microscope, Journal of Structural Biology, volume 155, , issue 1, pp 63-73, July 2006

2) Stokes et al, A New Approach to Studying Biological and Soft Materials Using Focused Ion Beam Scanning Electron Microscopy (FIBSEM), Journal of Physics: Conference Series 26, (2006) 50-53

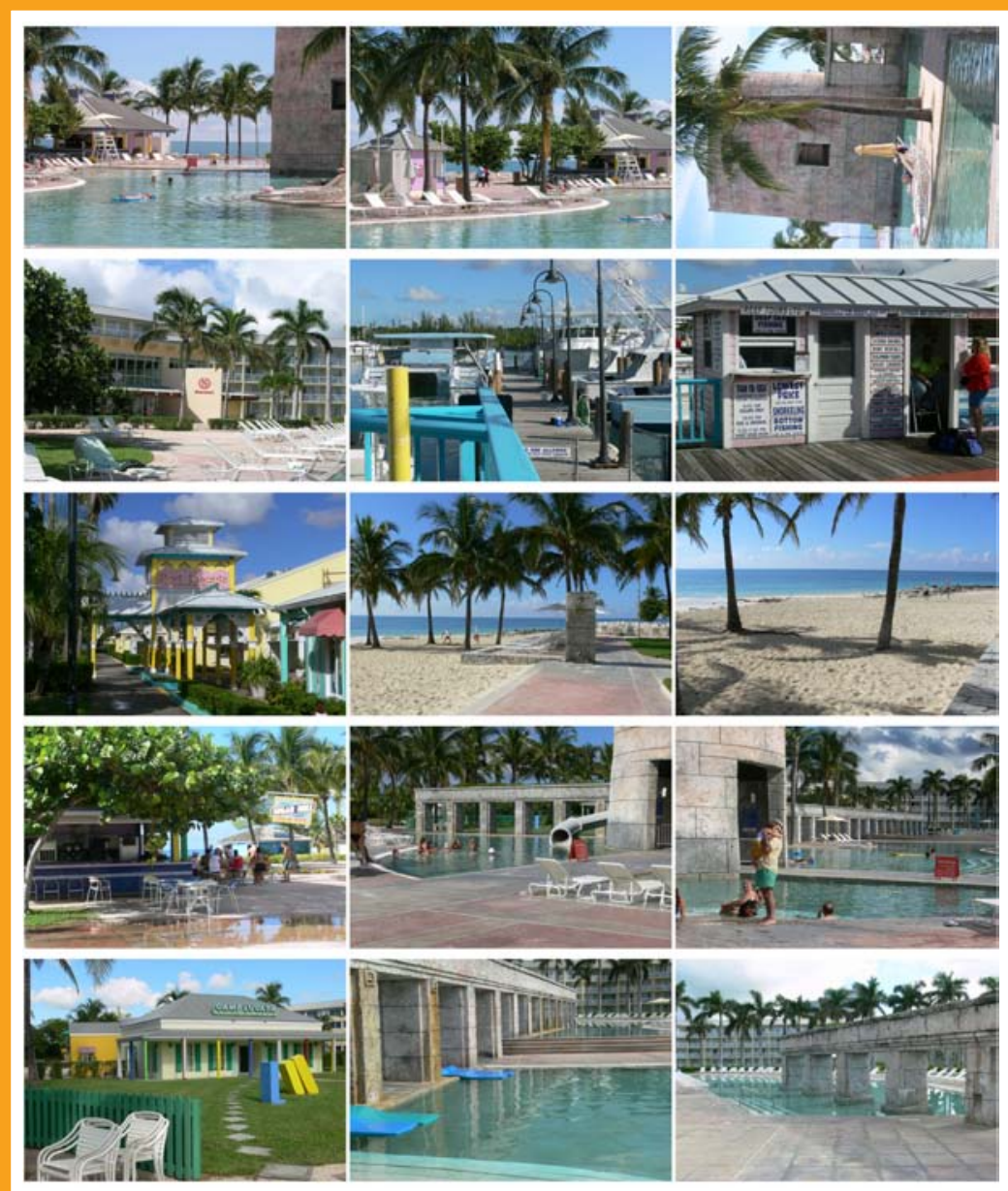

\section{VISITE GHE BAHAMRS!}

The Microscopy and Microanalysis - 2007 Local Arrangements Committee has arranged for a special rebate for attendees interested in same-day or overnight cruise packages to the Bahamas in the periods immedately before and after the M\&M-2007 meeting in Fort Lauderdale. Ship leaves from a pier adjacent to the Convention Center! Pictures on the left taken at one of the package hotels. There is a casino in this hotel complex.

More info and reservations at:

http://www.discoverycruise.com/microscopy

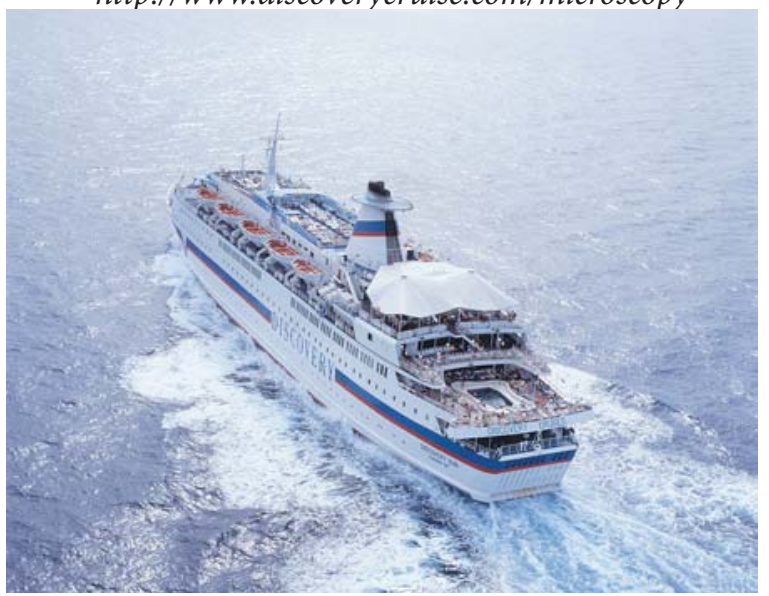




\section{Value and Excellence in SEMs}

\section{VEGA Scanning Electron Microscopes}

Fully automated PC-controlled SEMs

designed for a multi-user environment.

High performance 4-lens optics provides high resolution, high depth of focus, extremely large field of view as well as optimum working conditions for any application.

High working vacuum and variable pressure operation up to $2000 \mathrm{~Pa}$.

Original patented Low Vacuum Secondary Detector for true secondary electron imaging under low vacuum.

Full range of precision-engineered specimen chambers and stages.

Sophisticated yet easy-to-use software for microscope control and image processing \&t evaluation.

Network operations with built-in remote control and diagnostics.

3D surface reconstruction using $3 \mathrm{D}$ beam technology.
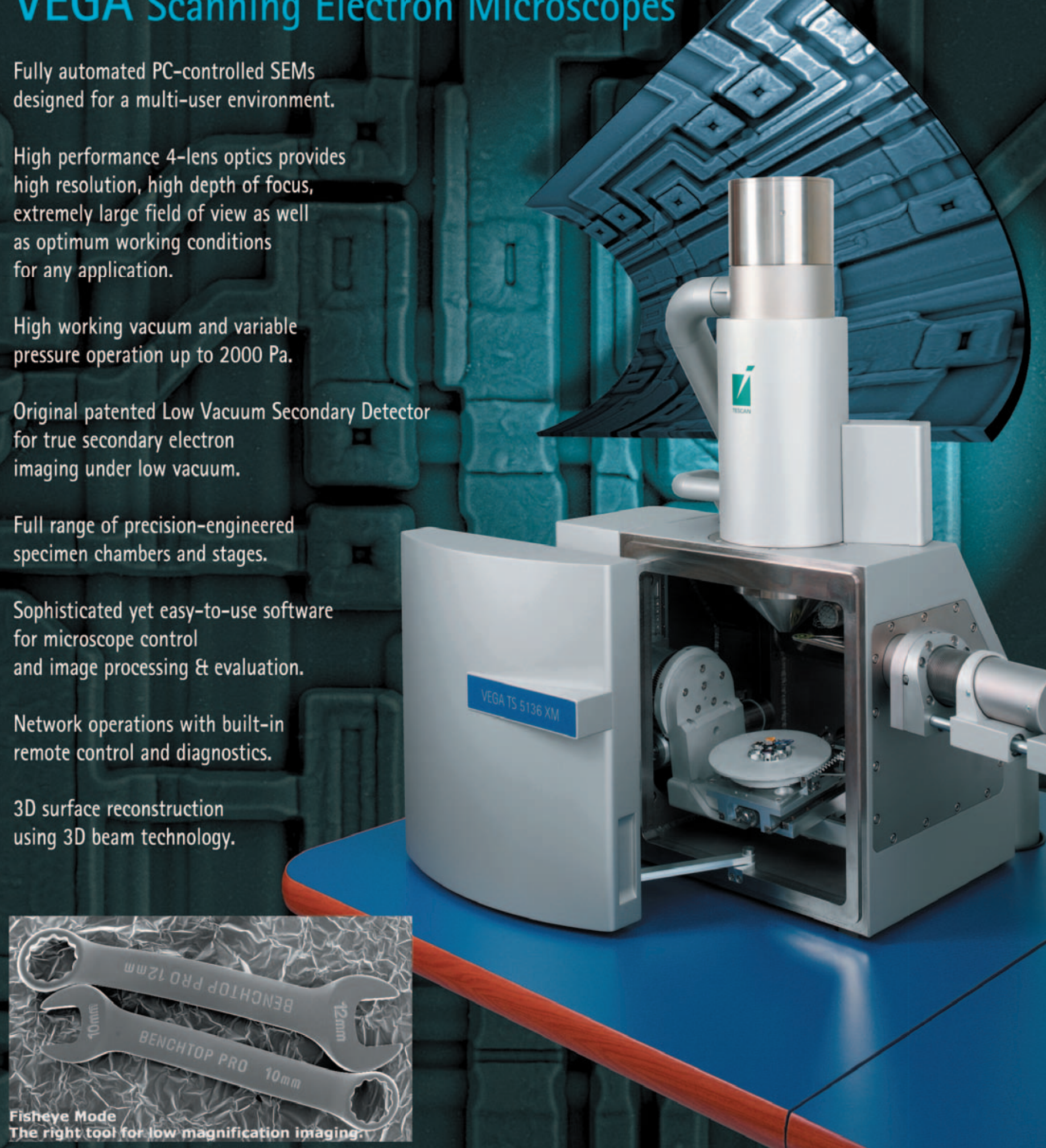

The right toof for tow magnification ipraging. I I

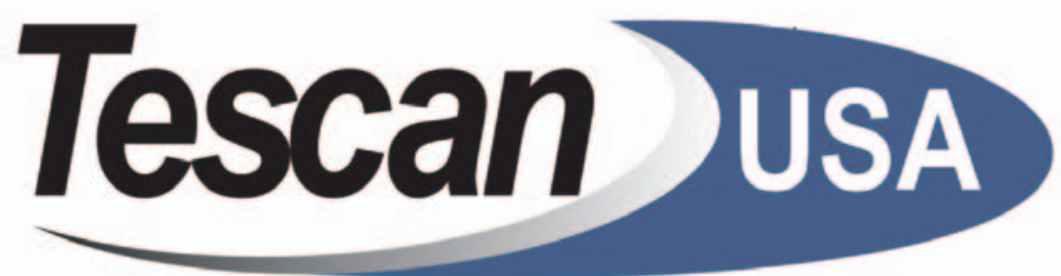

tel. 724-772-7433 www.tescan-usa.com 